\title{
OPEN Distinct soil bacterial patterns along narrow and broad elevational gradients in the grassland of Mt. Tianshan, China
}

\begin{abstract}
Rui Li ${ }^{1,2}$, Yunhua Liu ${ }^{1,2}$, Junhui Cheng ${ }^{1,2}$, Nana Xue ${ }^{1,2}$, Zongjiu Sun ${ }^{2}$, Pan Zhang ${ }^{1,2}$, Ning $\mathrm{Li}^{2}$, Xiaoshuang $\mathrm{Di}^{2}$, Weihua $\mathrm{Fan}^{2}$, Jiang Deng ${ }^{2}$, Yucheng $\mathrm{Ma}^{2}$, Minfei $\mathrm{Li}^{2}$ \& Jiandong Sheng ${ }^{1,2 \bowtie}$

Bacteria are essential regulators of soil biogeochemical cycles. While several studies of bacterial elevational patterns have been performed in recent years, the drivers of these patterns remain incompletely understood. To clarify bacterial distribution patterns and diversity across narrow- and broad-scale elevational gradients, we collected soil samples from 22 sites in the grasslands of $\mathrm{Mt}$. Tianshan in China along three elevational transects and the overall elevation transect: (1) 6 sites at elevations of 1047-1587 m, (2) 8 sites at 876-3070 m, and (3) 8 sites at 1602-2110 m. The bacterial community diversity across the overall elevation transects exhibited a hump-like pattern, whereas consistent patterns were not observed in the separate elevational transects. The bacterial community composition at the phylum level differed across the transects and elevation sites. The Actinobacteria was the most abundant phylum overall (41.76\%) but showed clear variations in the different transects. Furthermore, heatmap analyses revealed that both $\mathrm{pH}$ and mean annual temperature (MAT) were significantly $(P<0.05)$ correlated with bacterial community composition as well as the dominant bacterial phyla, classes, and genera. These findings provide an inclusive view of bacterial community structures in relation to the environmental factors of the different elevational patterns.
\end{abstract}

Elevational gradients have been studied to evaluate the relationships between altitude, spatial distribution patterns, and basic biome ecological processes in montane ecosystems ${ }^{1-3}$. Understanding how soil bacterial communities respond to particular environmental variables is of great ecological importance, and our current understanding of soil bacterial community elevational patterns in mountain grasslands remains relatively limited. There is thus a clear need for further research regarding bacterial elevational diversity and community composition patterns in mountain grassland ecosystems.

To date, several studies have described the patterns and drivers of soil bacterial community structures across elevational gradients in different montane regions ${ }^{4-7}$. For example, some studies have reported a hump-shaped pattern when evaluating soil bacterial community diversity as a function of elevation, as in a study conducted on Mount Fuji, evaluating soil bacterial diversity between 1000 and 3700 m, with a significant "peak" in bacterial diversity being detected at an elevation of approximately $2500 \mathrm{~m}^{8}$. Similarly found that microbes followed Humboldt diversity patterns between the elevations of $194 \mathrm{~m}$ and $3644 \mathrm{~m}^{6}$. However, some reports detected no clear patterns in soil bacterial community diversity along elevation gradients ${ }^{5,9}$, although decreases in soil Acidobacterial diversity have been observed as a function of elevation between 2460 and $3380 \mathrm{~m}^{4}$. Together, these findings suggest that bacterial composition and diversity respond differently to elevational gradients and environmental types, with bacterial communities, in particular, likely to exhibit a range of tolerance levels across different environmental conditions.

Meanwhile, bacterial community composition and diversity are known to vary substantially across montane spatial scales, and this variation is thought to be associated with changes in particular biotic or abiotic factors. The specific impacts of climatic, plant, and soil properties on bacterial community composition and diversity have been discussed in a range of studies. One widely accepted determinant of soil bacterial community distribution along elevation gradients in a range of mountain ecosystems is the soil $\mathrm{pH}^{9-12}$, although other studies have also suggested that other driver factors such as temperature may also be major regulators of bacterial

${ }^{1}$ College of Grassland and Environmental Sciences, Xinjiang Agricultural University, 311 Nongda East Road, Urumqi 830052, Xinjiang, China. ${ }^{2}$ Key Laboratory of Soil and Plant Ecological Processes of Xinjiang, Urumqi 830052, Xinjiang, China. ${ }^{\circledR}$ email: sjd@xjau.edu.cn 


\begin{tabular}{|c|c|c|c|c|c|c|c|}
\hline \multirow[b]{2}{*}{ Elevations (m) } & \multirow[b]{2}{*}{ pH } & \multicolumn{4}{|c|}{ Soil physicochemical parameters } & \multicolumn{2}{|c|}{ Plant parameters } \\
\hline & & SM (\%) & $\operatorname{SOC}\left(\mathrm{g} \mathrm{kg}^{-1}\right)$ & $\mathrm{TN}\left(\mathrm{g} \mathrm{kg}^{-1}\right)$ & C:N ratio & PSR $\left(\right.$ per $\left.\mathrm{m}^{-2}\right)$ & BGB $\left(\mathrm{g} \mathrm{m}^{-2}\right)$ \\
\hline 1047 & $8.45 \mathrm{a}$ & $1.20 \mathrm{~d}$ & $12.83 \mathrm{~d}$ & $1.02 \mathrm{~d}$ & $23.80 \mathrm{a}$ & $7.67 \mathrm{c}$ & $1106 \mathrm{bc}$ \\
\hline 1071 & $7.82 \mathrm{~b}$ & $2.12 \mathrm{~d}$ & $30.27 \mathrm{c}$ & $2.83 \mathrm{c}$ & $10.48 \mathrm{~b}$ & $11.00 \mathrm{~b}$ & $1086 c$ \\
\hline 1277 & $7.11 \mathrm{c}$ & $3.57 \mathrm{c}$ & $43.15 \mathrm{~b}$ & $4.11 \mathrm{~b}$ & $10.89 \mathrm{~b}$ & $9.00 \mathrm{bc}$ & $1011 \mathrm{c}$ \\
\hline 1423 & $8.29 \mathrm{a}$ & $3.29 \mathrm{c}$ & $12.83 \mathrm{~d}$ & $1.49 \mathrm{~d}$ & $12.14 \mathrm{~b}$ & $8.00 \mathrm{c}$ & $2165 \mathrm{~b}$ \\
\hline 1580 & $7.85 \mathrm{~b}$ & $10.46 \mathrm{~b}$ & $59.64 a$ & $4.69 \mathrm{~b}$ & $12.83 \mathrm{~b}$ & $15.00 \mathrm{a}$ & $3103 a$ \\
\hline 1587 & $7.65 \mathrm{~b}$ & $12.73 \mathrm{a}$ & $63.97 \mathrm{a}$ & $6.19 \mathrm{a}$ & $10.74 \mathrm{~b}$ & $15.33 \mathrm{a}$ & $2357 \mathrm{a}$ \\
\hline Transect 1 & 7.86 & 5.56 & 37.12 & 3.39 & 13.48 & 11.00 & 1805 \\
\hline 876 & $8.83 a$ & $1.21 \mathrm{~b}$ & $5.81 \mathrm{e}$ & $0.99 \mathrm{c}$ & $20.57 \mathrm{a}$ & $2.67 \mathrm{c}$ & $627 \mathrm{e}$ \\
\hline 920 & 8.44ab & $1.63 \mathrm{~b}$ & $9.51 \mathrm{e}$ & $1.20 \mathrm{c}$ & $19.94 \mathrm{a}$ & $5.67 \mathrm{~d}$ & 743de \\
\hline 1586 & $7.79 \mathrm{~b}$ & $4.04 \mathrm{~b}$ & $25.80 \mathrm{de}$ & $2.73 \mathrm{bc}$ & $11.45 \mathrm{c}$ & $4.33 \mathrm{~cd}$ & $1192 \mathrm{~cd}$ \\
\hline 1744 & $6.55 c$ & 20.29 & $85.92 \mathrm{ab}$ & $6.37 \mathrm{a}$ & $16.23 \mathrm{~b}$ & $8.67 \mathrm{~b}$ & 3099a \\
\hline 2513 & 6.14cd & 17.16 & $65.47 \mathrm{bc}$ & $6.47 \mathrm{a}$ & $10.48 \mathrm{c}$ & $9.00 \mathrm{~b}$ & $1373 b c$ \\
\hline 2903 & 5.78cde & 23.51 & \begin{tabular}{|l|}
$100.66 a$ \\
\end{tabular} & $6.51 \mathrm{a}$ & $18.97 \mathrm{a}$ & $12.00 \mathrm{a}$ & $3427 \mathrm{a}$ \\
\hline 2981 & $5.02 \mathrm{e}$ & 25.88 & $47.70 \mathrm{~cd}$ & $4.34 \mathrm{ab}$ & $10.72 \mathrm{c}$ & $13.67 \mathrm{a}$ & $3466 a$ \\
\hline 3070 & 5.29de & 15.46 & $43.92 \mathrm{~cd}$ & $2.87 \mathrm{bc}$ & $15.29 \mathrm{~b}$ & $9.00 \mathrm{~b}$ & $1725 b$ \\
\hline Transect 2 & 6.73 & 13.65 & 48.10 & 3.94 & 15.46 & 8.13 & 1957 \\
\hline 1602 & $7.82 \mathrm{a}$ & 9.19d & $37.87 \mathrm{c}$ & $3.79 \mathrm{~d}$ & $10.83 \mathrm{~d}$ & $14.67 \mathrm{bc}$ & $2221 b c$ \\
\hline 1661 & 7.64ab & $19.66 \mathrm{c}$ & $38.10 \mathrm{c}$ & $2.83 \mathrm{~d}$ & $19.77 \mathrm{a}$ & $7.33 \mathrm{e}$ & 1374de \\
\hline 1703 & $6.90 \mathrm{~b}$ & $19.53 c$ & 110.38ab & $10.63 \mathrm{a}$ & $10.57 \mathrm{~d}$ & $14.33 \mathrm{~cd}$ & $1467 \mathrm{de}$ \\
\hline 1739 & $6.94 \mathrm{~b}$ & $19.46 c$ & $92.47 \mathrm{~b}$ & $9.07 \mathrm{ab}$ & $10.70 \mathrm{~d}$ & $11.33 \mathrm{~d}$ & $1663 \mathrm{~cd}$ \\
\hline 1998 & 7.40ab & $8.40 \mathrm{~d}$ & $48.84 \mathrm{c}$ & $4.24 \mathrm{~d}$ & $14.45 \mathrm{c}$ & $11.67 \mathrm{~cd}$ & $862 \mathrm{e}$ \\
\hline 2045 & $5.94 \mathrm{c}$ & $35.14 \mathrm{a}$ & 113.00ab & $10.53 a$ & $10.85 \mathrm{~d}$ & $24.00 \mathrm{a}$ & $3029 \mathrm{a}$ \\
\hline 2075 & $5.99 \mathrm{c}$ & $26.54 \mathrm{~b}$ & $94.29 \mathrm{~b}$ & $6.18 \mathrm{c}$ & $15.43 \mathrm{bc}$ & $22.33 \mathrm{a}$ & $2020 \mathrm{~cd}$ \\
\hline 2110 & $5.54 \mathrm{c}$ & $30.07 \mathrm{~b}$ & $123.74 \mathrm{a}$ & $7.44 \mathrm{bc}$ & $16.88 \mathrm{~b}$ & $19.33 b$ & $2827 \mathrm{ab}$ \\
\hline Transect 3 & \begin{tabular}{|l|}
6.77 \\
\end{tabular} & 21.00 & \begin{tabular}{|l|}
82.34 \\
\end{tabular} & 6.84 & 13.69 & 15.62 & 1933 \\
\hline
\end{tabular}

Table 1. Soil properties and plant variables at different sampled elevations. SM soil moisture, SOC soil organic carbon, $T N$ total nitrogen, PSR plant species richness, $B G B$ belowground biomass. Values in bold indicate the mean value in the indicated Transects.

community composition ${ }^{6,13,14}$. For example, studies conducted on Changbai Mountain ${ }^{9}$ and Segrila Mountain ${ }^{12}$ in China found that soil $\mathrm{pH}$ had a major influence on bacterial elevational patterns, whereas a separate study of an Andean transect traversing a $3.5 \mathrm{~km}$ elevation range determined temperature to be the predominant driver of bacterial distribution patterns ${ }^{6}$. Therefore, soil bacterial patterns may thus be dependent on the study scale, and the environmental stress gradient hypothesis outlines the key response strategies of soil bacteria when faced with environmental change.

Mt. Tianshan is one of the seven major mountain systems in the world, covering a large region of the Eurasian hinterland. Roughly two-thirds of the mountains in this range are located in the Xinjiang Uygur Autonomous Region of China. Besides, Mt Tianshan grassland is characterized by a typical arid and semiarid continental climate, as well as having a clear vertical zonation from desert to alpine meadow biomes. However, there is little knowledge of the shaping of bacterial communities across elevational gradients in these special habitats. In addition, several questions have emerged concerning in the patterns, drivers, and biogeographical differences of soil communities in Mt Tianshan grassland. Are there any differences in the soil bacterial communities in different areas of Mt Tianshan grassland, for instance, in different administrative regions? Due to the known impact of environmental factors in shaping of soil bacterial communities across montane spatial scales, does this relationship also exist in Mt Tianshan grassland? and, if so, what is the key factor?

To answer these questions, three typical administrative regions (transects 1,2 , and 3 ) were selected to study (1) the distribution patterns of soil bacteria in mountain grassland ecosystems; (2) the biogeographical differences in soil communities in the Mt Tianshan grassland; (3) the influence of environmental disturbance in shaping bacterial community diversity along the elevation gradients.

\section{Results}

Environmental variable quantification along an altitudinal gradient. This study area included 22 sampling sites, and 66 samples, classified into three transects, namely Transect 1 (1047-1587 m), Transect $2(876-3070 \mathrm{~m})$, and Transect $3(1602-2110 \mathrm{~m})$. Significant differences in soil properties and plant parameters were observed along the three studied altitudinal transects $(P<0.05)$ (Table 1$)$. The soil $\mathrm{pH}$ ranged from 7.11 to $8.45,5.54$ to 7.82 , and 5.02 to 8.83 in Transects 1,2 , and 3, respectively. In contrast to altitudinal Transects 1 and 3, the broad-scale altitudinal gradients of Transect 2(876-3070 m) exhibited more substantial changes in soil moisture (SM), soil organic carbon (SOC), total nitrogen (TN), C:N ratio $(\mathrm{C} / \mathrm{N})$, plant species richness (PSR), and belowground biomass (BGB) between sampling sites. Compared to sites situated at $876 \mathrm{~m}, 920 \mathrm{~m}$, 
and $3070 \mathrm{~m}$, the intermediate altitude site samples at $1744 \mathrm{~m}$ and $2513 \mathrm{~m}$ tended towards increased SM, TN, and SOC. In contrast, PSR and BGB were the highest in the high-altitude alpine meadow environments at $2903 \mathrm{~m}$ and $2981 \mathrm{~m}$.

Bacterial community composition across the altitudinal gradients. Over the study area, the Actinobacteria constituted the most abundant phylum in all 22 sampling sites on Mt. Tianshan, accounting for $41.76 \%$ of total sequences, followed by the Proteobacteria (24.13\%), Acidobacteria (10.85\%), Chloroflexi (8.73\%), Gemmatimonadetes (3.65\%), Verrucomicrobia (3.23\%), Bacteroidetes (1.64\%) and Planctomycetes (1.53\%) (Fig. 1A). Although there were consistent trends in soil bacterial phylum composition across samples the average relative abundance of phyla varied across elevations. For example, in Transect $2(876-3070 \mathrm{~m})$, the relative Actinobacteria abundance was lower in the high elevation sites $(2903 \mathrm{~m}$ and $2981 \mathrm{~m})$ relative to both the low $(876 \mathrm{~m}$ and $920 \mathrm{~m})$ and the intermediate elevation sites $(1744 \mathrm{~m}$ and $2513 \mathrm{~m})$. At the class level, a total of 11 classes were detected, with 10 having a relative abundance $>0.05 \%$, while the remaining bacteria were merged into an "others" class. As shown in Fig. 1B, the proportion of Actinobacteria, Alphaproteobacteria and Gammaproteobacteria at each elevation was 45\%, whereas Deltaproteabacteria, Acidobacteria_Subgroup_6, and Gemmatimonadetes were prevalent at low levels in most soil samples. At the genus level (Supplementary Fig. 1 ), 76 genera were detected in the research areas, with the dominant genera including norank_f_67-14_o_Solirubrobacterales (5.72\%), Rubrobacter (4.35\%), Solirubrobacter (2.83\%), Pseudonocardia (2.26\%) and Bradyrhizobium $(2.19 \%)$ and less than $0.01 \%$ of the bacterial genera were classified into others.

Bacterial community composition varies along elevation gradients. We next sought to analyze the differences in relative bacterial abundance at the phylum level among Transects 1-3 (Fig. 2). Significant differences in the relative abundance of Actinobacteria, Proteobacteria, Acidobacteria, Verrucomicrobia, Firmicutes, and Rokubacteria were detected in samples from the different transects (Fig. 2A). The relative abundance of Actinobacteria and Firmicutes in Transect 1 (48.64\% and 1.89\%, respectively) was significantly higher than in Transect 2 (38.43\% and $1.49 \%$, respectively) and Transect $3(39.63 \%$ and $0.98 \%$, respectively) $(P<0.001$ for all). In contrast, relative abundance of Acidobacteria and Verrucomicrobia in Transect 1 was lower $(8.54 \%$ and $1.51 \%$, respectively) than in Transect $2(11.48 \%$ and $4.38 \%$, respectively) and Transect $3(12.06 \%$ and $3.37 \%$, respectively) $(P<0.001$ for all).

The relative abundance of the dominant phyla in the bacterial communities did not differ significantly among the six elevation sites (S1047, S1071, S1277, S1423, S1580, and S1587) in Transect 1, whereas significant changes were observed in the Transect 2 and Transect 3 elevation sites. The relative Proteobacteria, Acidobacteria and Verrucomicrobia abundance at the high elevation sites in Transect 2 and 3 (i.e., S2903 and S2981 in Transect 2, S2075 and S2110 in Transect 3) were significantly higher than those at low elevation sites in both transects (S876 and S920 in Transect 2 and S1602 and S1661 in Transect 3), whereas the opposite trend was observed for Actinobacteria and Gemmatimonadetes.

Bacterial alpha diversity elevation patterns. A total of 3,335,681 quality sequences across all 66 soil samples were grouped into 5314 operational taxonomic units (OTUs) at a 97\% similarity level. We identified significant relationships between soil bacterial richness, diversity, and elevation (Fig. 3). No dramatic changes in the elevational bacterial diversity patterns were observed between Transects 1, 2 and 3 (Fig. 3A-C,E-G). However, across the overall elevational gradient, the Chaol and Shannon indices exhibited pronounced hump-shaped patterns (Fig. 3D,H).

The "peak" value in Chaol richness was detected at lower elevations at around $1423 \mathrm{~m}$, while a "hollow" pattern was evident at an intermediate elevation of $2513 \mathrm{~m}$ (Fig. 3D). Shannon diversity decreased from 876 to $2513 \mathrm{~m}$ (Fig. 3H), but increased from 2513 to $3070 \mathrm{~m}$. These data suggest that soil bacterial community diversity conformed to a hump-shaped profile in the Mt. Tianshan grasslands.

Bacterial community beta diversity along elevational gradients. A nonmetric multidimensional scaling analysis (NMDS) was next conducted to evaluate overall bacterial community structure composition at the OTU level. As shown in Fig. 4A, two small-scale elevational samples (1047-1587 m and 1602-2110 m) were significantly separated from one another (ANOSIM test, stress $=0.0104, \mathrm{R}=0.19, P=0.001$ ), and the elliptical coverage of the broader-scale elevation samples (Transect 2, 876-3070 m) partially overlapped with that of these two small-scale elevation samples (Transect 1, 1047-1587 $\mathrm{m}$ and Transect 3, 1602-2110 m). Overall, there were significant changes in the bacterial community structure in the three analyzed transects (Fig. 4B-D), except for several neighboring sites in the 1602-2110 m elevation range (S1703, S1739, S2045, S2075, and S2110). This suggests that elevation had the most significant impact on bacterial community structure in the Mt. Tianshan grassland.

Relationships between bacterial community structures and environmental variables. Lastly, we evaluated correlations between environmental variables and the top 30 soil bacterial phyla, classes and genera using heatmaps, revealing that the soil bacterial community composition was simultaneously affected by climatic, plant, and soil physicochemical factors (Fig. 5; Supplementary Fig. 2). The relationships between the environmental variables and relative abundance varied as a function of bacterial phylum and class which are affected differently by environmental factors. As shown in Fig. 5, Spearman correlation analysis revealed that soil $\mathrm{pH}$ and mean annual temperature (MAT) were the key factors influencing on the relative abundances of the main bacterial phyla and classes. The abundance of Actinomycetes, Gemmatimonadetes and Chloroflexi phyla, as well as the diversity variables, were significantly and positively correlated with both soil $\mathrm{pH}$ and PET (Fig. 5A; 
A

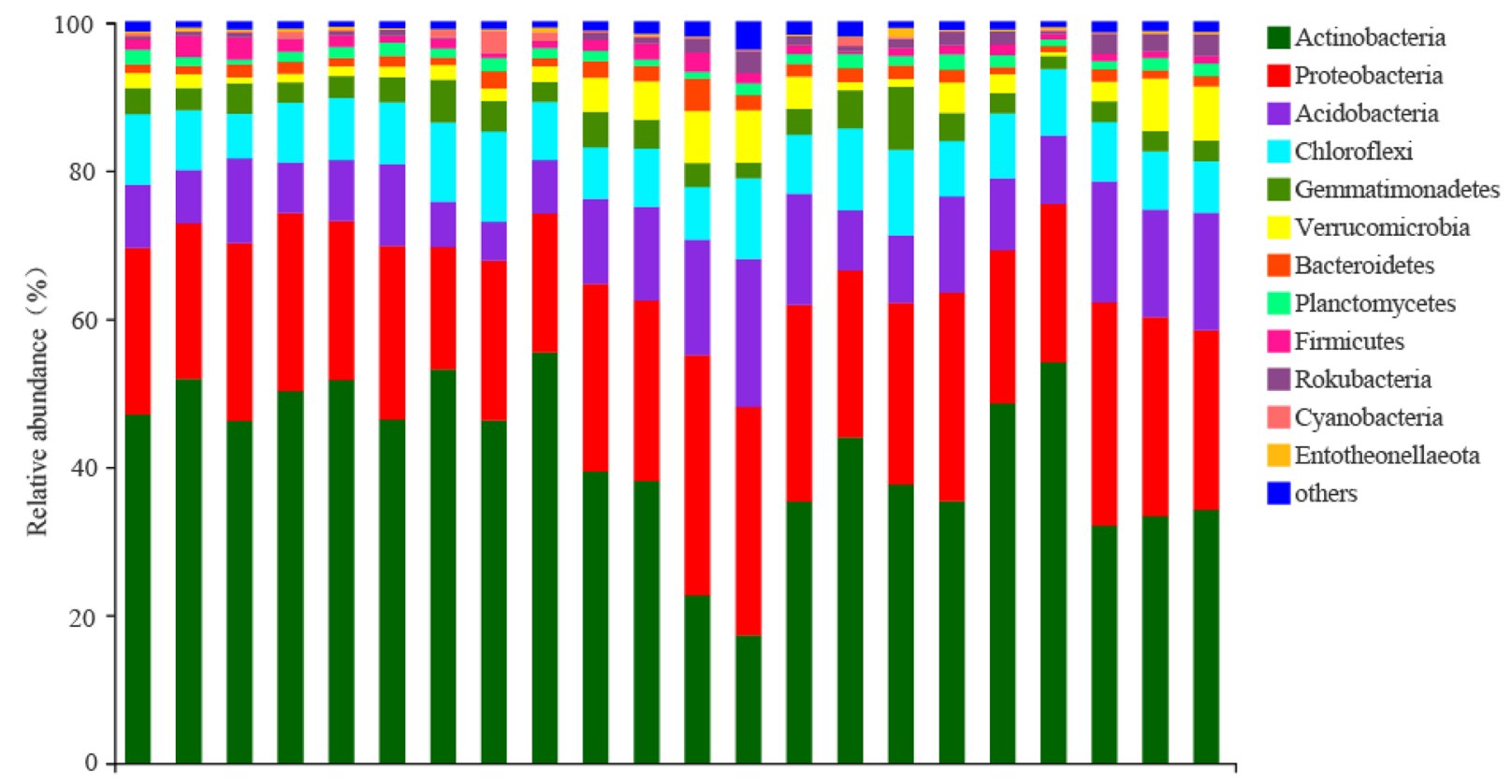

B

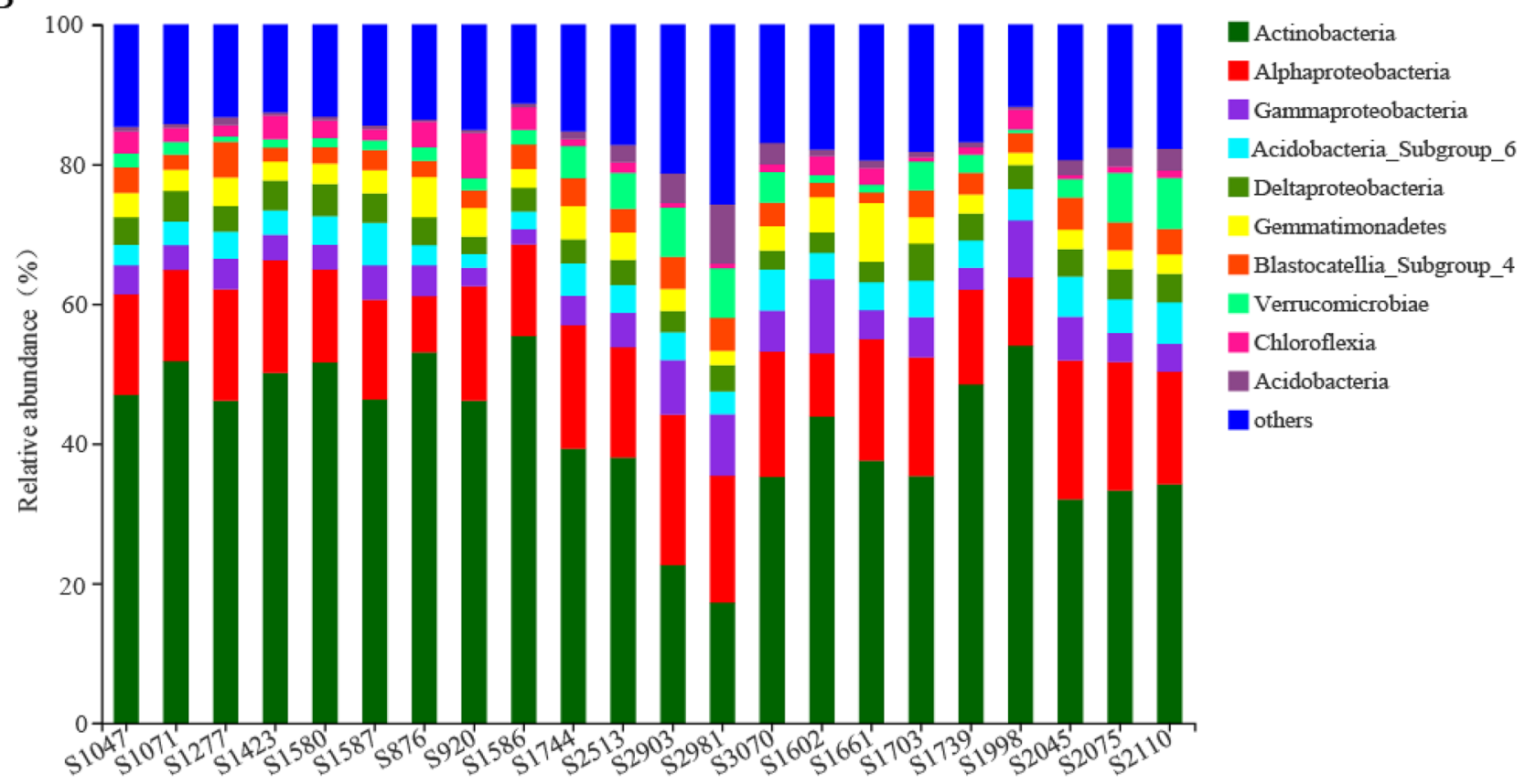

Transect 1

Transect 2

Transect 3

Elevation $(\mathrm{m})$

Figure 1. Bacterial community composition variations at the phylum (A) and class (B) levels in soil samples collected at different levels. These were done in R (v3.3.1, http://www.R-project.org).

Table 2; Supplementary Fig. 2; $P<0.001$ ), while Acidobacteria, Proteobacteria and Verrucomicrobia showed significant negative correlations with soil $\mathrm{pH}$ and (potential evapotranspiration) PET. Additionally, at the genus level, MAT, PET, and pH were also significantly correlated with bacterial genera (Supplementary Fig. 2). 
A

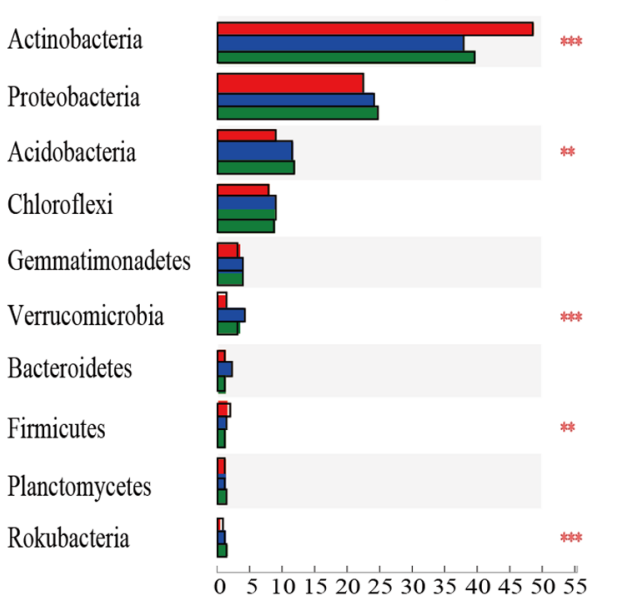

Relative abundance ( \% )

(1)

(n)

- Transect 1

- Transect 2

Transect 3

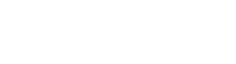

\section{C}

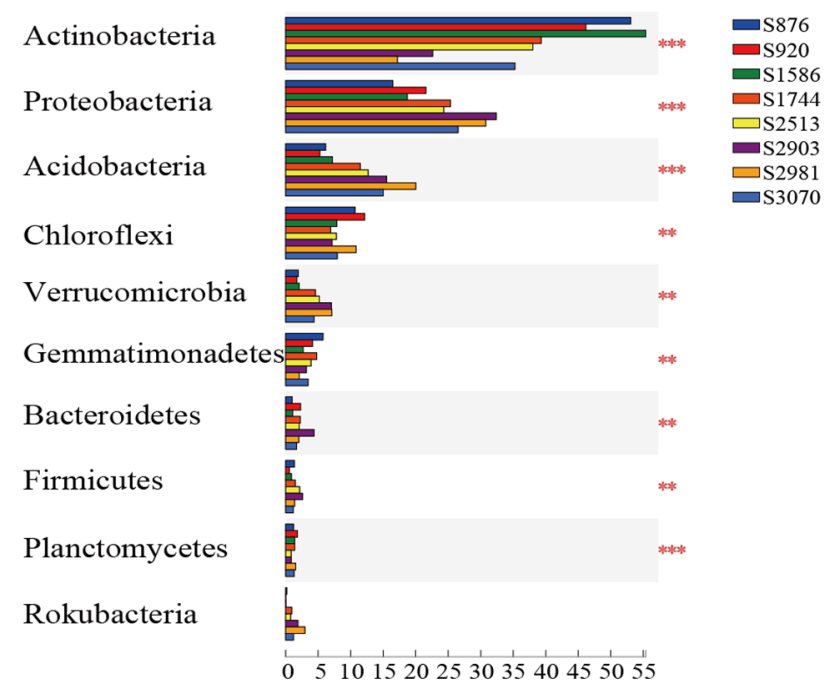

Relative abundance ( \%)
B

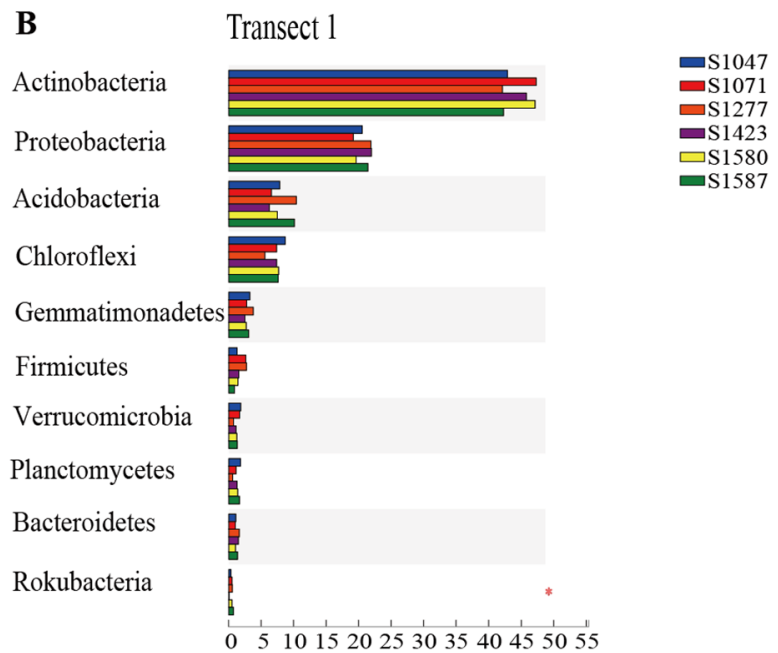

Relative abundance ( $\%$ )

D

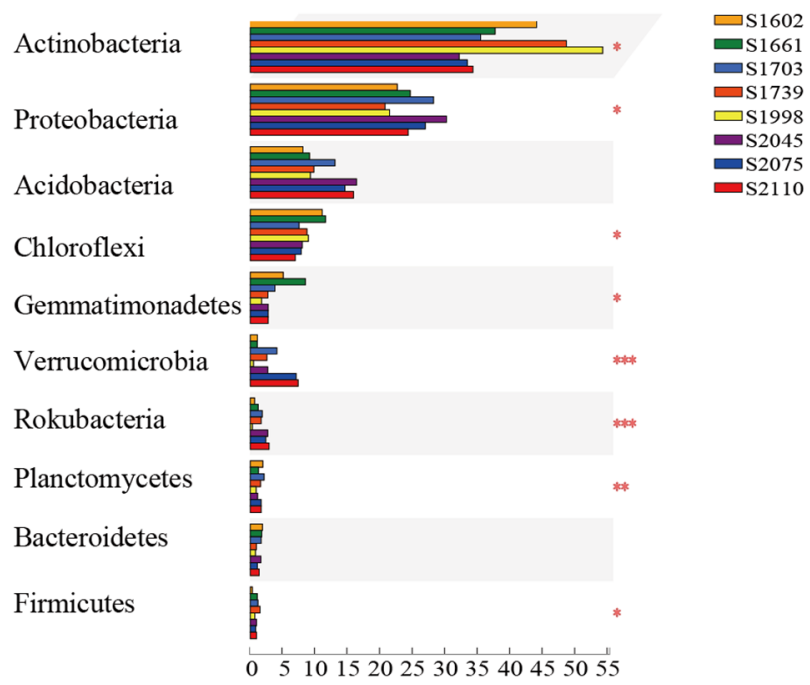

Relative abundance ( $\%$ )

Figure 2. Bacterial community composition variations at the phylum level in different Transects and elevation sites. The vertical axis denotes phylum name, while the horizontal axis represents the average relative abundance in samples from different elevations, and differently colored columns represent different elevation sites. $P$ values (one-way ANOVA) are marked on the right side of the graph, ${ }^{\star} P<0.05,{ }^{* *} P<0.01,{ }^{* *} P<0.001$. These were done in R (v3.3.1, http://www.R-project.org).

\section{Discussion}

Elevation patterns of bacterial community. In this study, we detected a hump-shaped richness/diversity pattern along a broad-scale elevation gradient (from 876 to $3070 \mathrm{~m}$ ) in the Mt. Tianshan grassland ecosystem, although consistent patterns were not observed along elevation gradients in the three analyzed transects. Other studies have also observed hollow-shaped bacterial community distribution patterns at higher elevations (1820-4050 m) on Laojun Mountain, China ${ }^{15}$, while a hump-shaped bacterial diversity pattern was observed at between elevations of 1000-3700 m on Mount Fuji in Japan ${ }^{8}$, with maximal diversity occurring at $2500 \mathrm{~m}$. In the present study, we observed maximal bacterial richness/diversity at $1277 \mathrm{~m}$, which may be attributable to differences in baseline environmental variables in these different ecosystems ${ }^{12}$. Other studies have reported no significant elevation-related trend $s^{5,7}$ or decreasing elevation patterns ${ }^{12}$. One study reported no apparent elevational pattern in bacterial diversity along a broad elevational gradient (530-2200 m) on Changbai Mountain, China, whereas, in a narrow gradient (2000-2500 m) above the $1950 \mathrm{~m}$ baseline, a monotonically decreasing elevation pattern was detected ${ }^{16}$. A U-shaped diversity elevation pattern was found under $1770 \mathrm{~m}$, whereas above this altitude a decreasing diversity pattern was observed with increasing elevation ${ }^{17}$. These prior findings underscore 

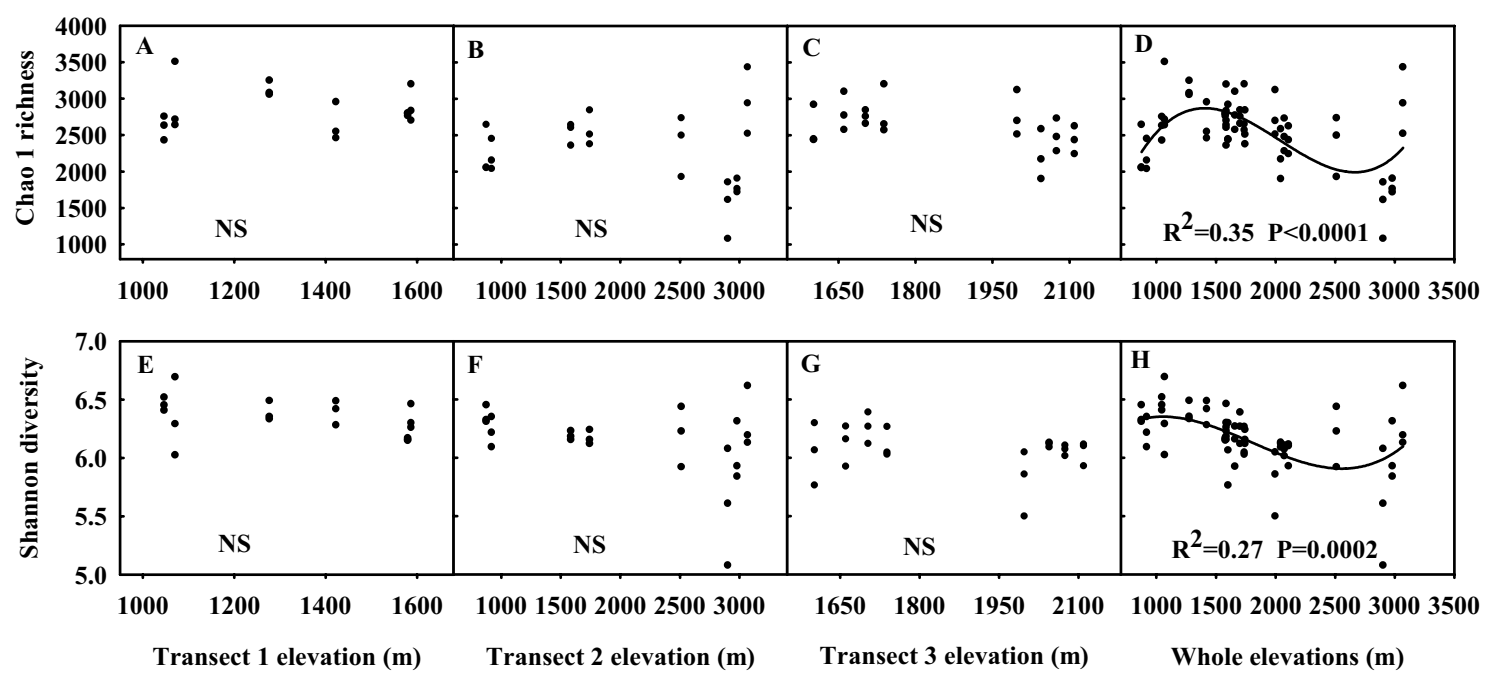

Figure 3. Relationships between bacteria alpha diversity and Transect 1 elevation gradients, Transect 2 elevation gradients, Transect 3 elevation gradients, and overall elevation gradients. Cubic models were tested to describe these relationships and model selection was conducted out based on $\mathrm{R}^{2}$ values and RMSE (root mean square error). $P$ values are given to indicate significance levels. NS not significant. Cubic models were used SigmaPlot v 10.0 (Systat Software, San Jose, CA).

the profound potential impact of study scales and sampling schemes on observed elevation patterns, showing the importance of using broader elevation scales when exploring bacterial community elevational patterns.

The choice of proper scale plays an important role in researching environmental variables and microbial community patterns. Narrow-scale elevational gradients provide a more precise description of the changes in bacterial community structures than broader scales. Nevertheless, changes in some phyla occur at specific altitudes, for example, in Transect 3 at S1739 and S1998 there was an increase in the relative abundance of Actinobacteria and a decrease in Proteobacteria and Acidobacteria. This was not detected in the broad scale Transect 2. In this environment, at the low altitudes of Transect 1 , it is not necessary to include a six-point of altitude scale because the differences in phyla abundance are not significant. Conversely, bacterial diversity needs broad altitudinal scales to show a consistent variation pattern. The scale (number of sampled points at different elevations) depends on each specific mountain environment and the specific altitude.

The exact factors responsible for the observed variability in elevation diversity patterns in these earlier studies remain to be clarified. We hypothesize that the use of broad elevation scales, as well as ecosystem-specific differences, may have contributed to these differences. The generality of these observed patterns across different habitats remains to be examined in future studies.

Environmental factors affecting bacterial community composition. In the present study, we observed a significant correlation between bacterial community composition and soil $\mathrm{pH}$, with MAT being the factor next most closely related to bacterial diversity (Fig. 6). In addition to elevation, soil pH, MAT, and MAP were all significantly correlated with bacterial diversity $(P<0.05$; Table 2$)$. Soil $\mathrm{pH}$ has been reported to be the strongest predictor of bacterial diversity along elevational gradients ${ }^{18}$. Consistent with this, Shen et al. ${ }^{9,12}$ found that $\mathrm{pH}$ plays the largest role in modulating bacterial community variations on Changbai Mountain. We additionally found a secondary role for MAT and belowground root biomass (BGB) as regulators of such diversity when soil $\mathrm{pH}$ was not constant. Nottingham et al. ${ }^{6}$ found MAT to be the primary determinant of bacterial elevational diversity patterns in situations with constant soil $\mathrm{pH}$. Temperature is often correlated with BGB and soil $\mathrm{pH}$, indirectly affecting microbial communities, thus potentially explaining these results ${ }^{19,20}$.

Elevational scale is an important factor to consider when interpreting the results of this and similar studies. Elevation has been reported to be indicative of bacterial community diversity in soil and sediment environments ${ }^{3,7,15,21}$. Sampling a broad soil $\mathrm{pH}$ range across study sampling sites is a prerequisite for detecting $\mathrm{pH}$-related differences in bacterial community compositions. If the soil $\mathrm{pH}$ range in this study had been narrower, MAT would likely have been the dominant determinant of relative bacterial abundance. Prior elevational studies on different mountains have reported soil $\mathrm{pH}$ ranges that were either wholly acidic or alkaline, whereas we found that the $\mathrm{pH}$ range in our study varied from acidic to alkaline (5.02-8.83), thus incorporating a broader range. Other environmental variables were also highly variable across study sites, likely reflecting the relatively complex conditions associated with these different elevations in general.

The analysis of bacterial composition revealed that Actinobacteria, Proteobacteria and Acidobacteria were the dominant phyla across the different transects and elevational sites on the Mt. Tianshan (Fig. 1), with significant differences in soil bacterial community composition between transects (Fig. 2). Changes in the bacterial community composition as a function of elevation have previously been thought to be attributable to habitat filtering as temperatures are lower at higher elevations ${ }^{15}$ and soil $\mathrm{pH}$ can vary across elevational study sites ${ }^{16}$. 
A The whole elevation transect

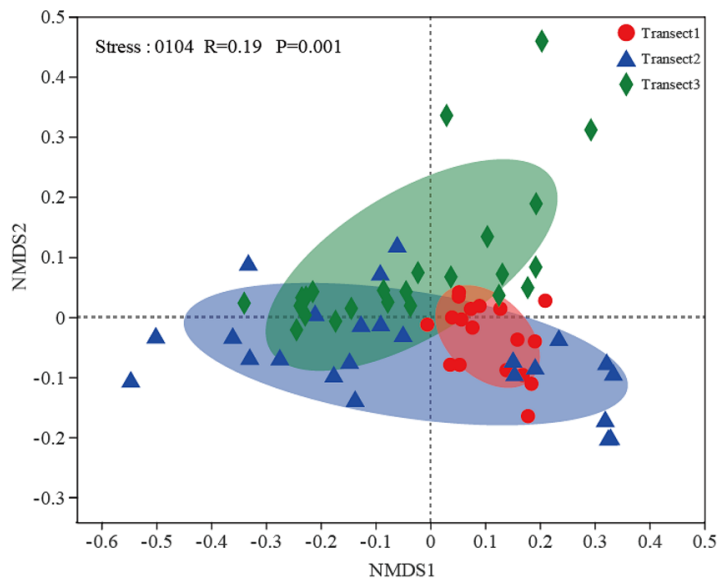

$\mathrm{C}$

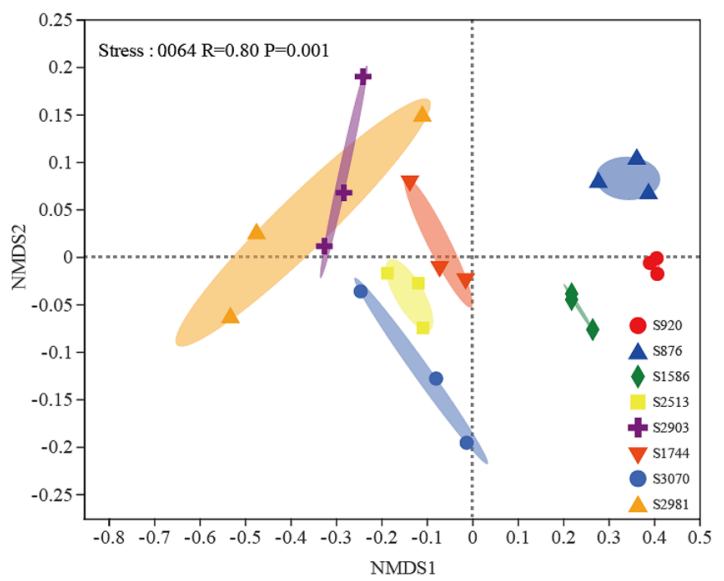

$\mathrm{B}$

Transect 1

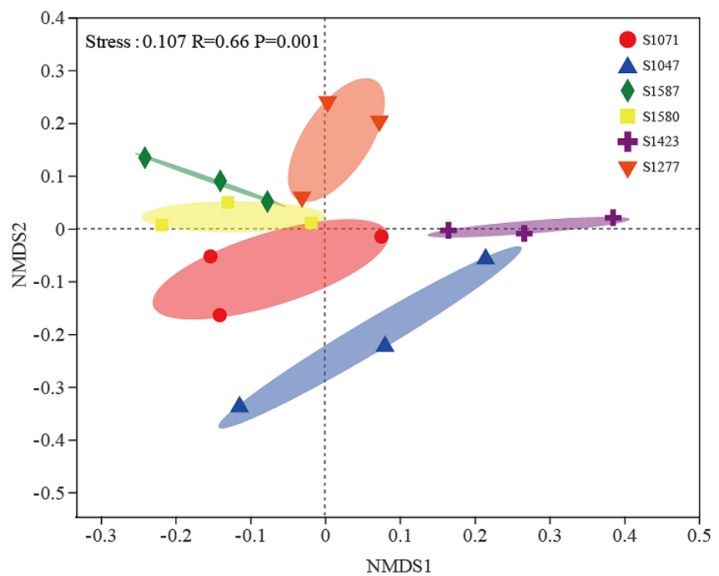

$\mathrm{D}$

Transect 3

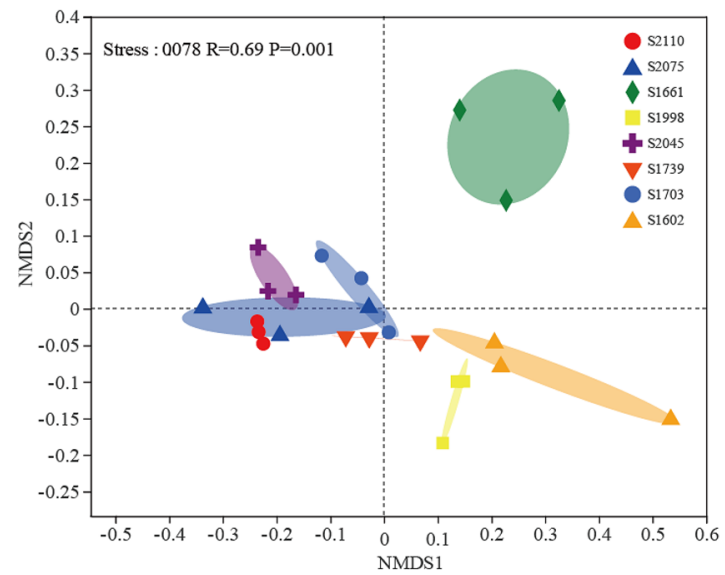

Figure 4. Nonmetric multidimensional scaling (NMDS) analysis of bacterial community composition as a function of elevation sites across all samples sites (A), elevational Transect 1 (B), elevational Transect 2 (C), and elevational Transect 3 (D). The NMDS analysis was performed on the Bray Curtis similarity matrix, calculated based upon total OTUs. To corroborate the NMDS results, a one-way ANOSIM (analysis of similarities) was used to test the relationship effects of elevation on bacterial community beta diversity. These were done in $\mathrm{R}$ with the vegan package (v3.3.1, http://www.R-project.org).

In this study, we found considerable variation in the climate, plant, and soil properties which all varied dramatically across the sampled sites (Tables 1; Supplementary Table 1). The relative abundances of the phyla Acidobacteria, Actinobacteria, Gemmatimonadetes, and Chloroflexi were significantly correlated with pH (Fig. 5), consistent with results from prior analyses of montane biomes conducted using horizontal ${ }^{10}$ (at the continental scale across North and South America) and vertical ${ }^{7}$ (100-1950 m on Mt. Halla, South Korea) research scales. These results were in line with those of studies conducted on the Changbai Mountain, such as the article published by Shen et al. ${ }^{9}$, which found that the Acidobacteria, Actinobacteria, Alphaproteobacteria, and Betaproteobacteria abundance varied as a function of elevation, and that alterations in community composition were significantly correlated with soil $\mathrm{pH}, \mathrm{C} / \mathrm{N}$, and moisture. In other studies, the Acidobacteria abundance has been shown to be significantly correlated with soil $\mathrm{pH}^{22}$, with these bacteria preferentially inhabiting neutral alkaline soil environments ${ }^{23}$. There are two likely explanations for why $\mathrm{pH}$ was the best predictor of bacterial community composition ${ }^{10}$. First, $\mathrm{pH}$ imposes direct physiological stress on bacterial growth ${ }^{24}$. Second, $\mathrm{pH}$ can also serve as an integrated functional index for other proximal factors that may influence local bacterial communities given that many different factors are directly or indirectly associated with soil $\mathrm{pH}^{21,24}$.

\section{Conclusion}

In this study, the altitudinal patterns of bacterial composition and diversity observed varied significantly with elevation scale in the mountain grassland of Mt. Tianshan. The bacterial diversity exhibited a hump-shaped pattern when evaluating the overall elevation gradient, whereas no consistent pattern was detected on a narrowerscale. While bacterial community elevation patterns were significantly responsive to mean annual temperature (MAT) and potential evapotranspiration (PET) throughout the entire elevation gradient, they were more strongly affected by soil $\mathrm{pH}$, which also influenced the elevational distribution pattern of soil bacterial diversity. The 
A Spearman Correlation Heatmap at Phylum Level

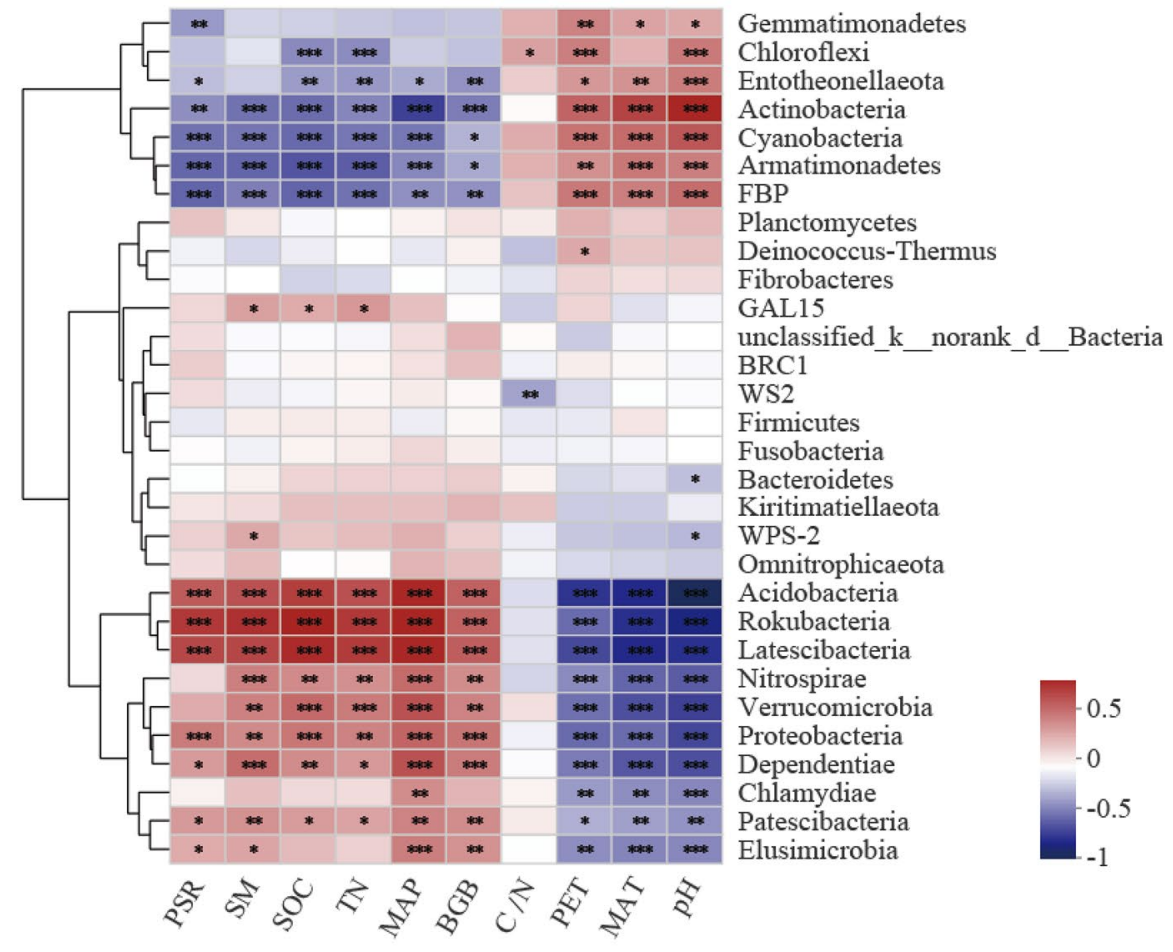

B

\section{Spearman Correlation Heatmap at Class Level}

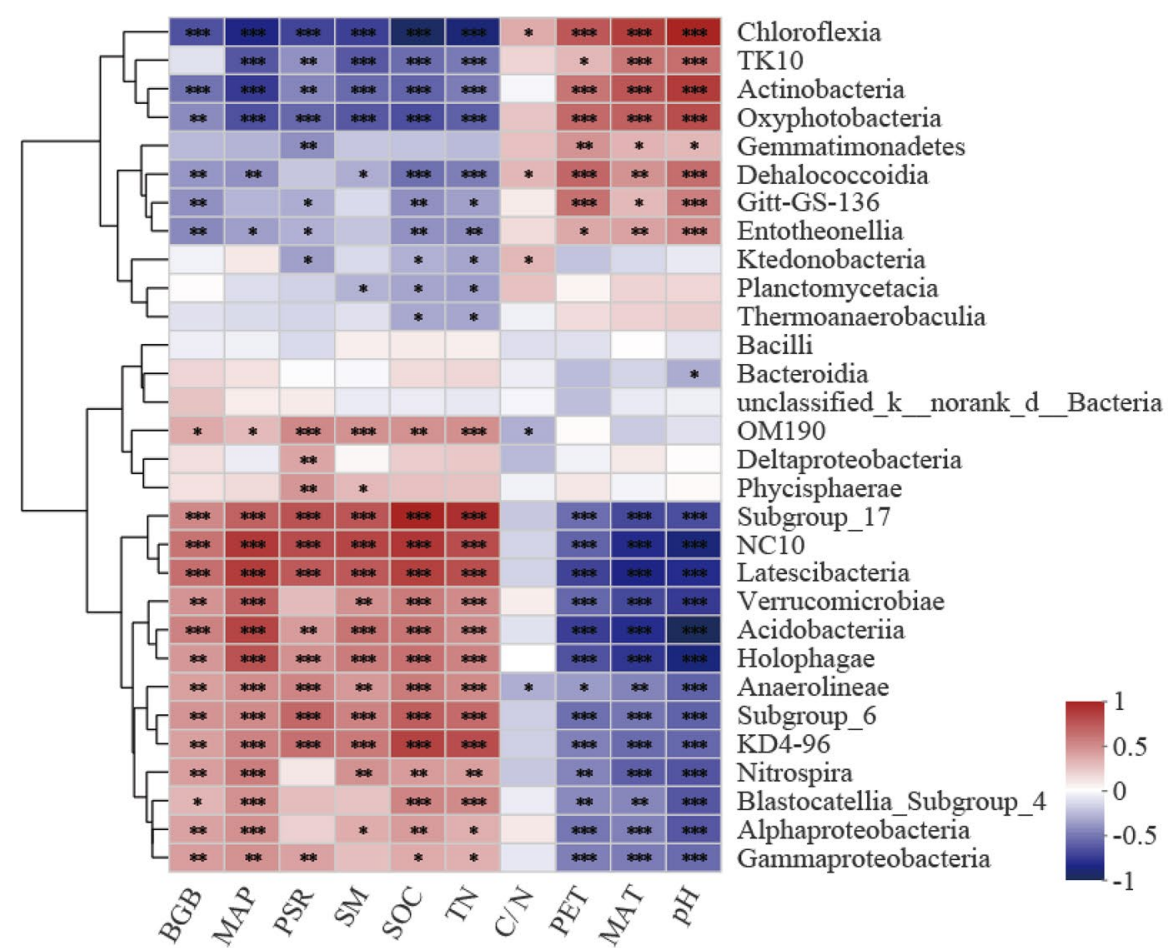

Figure 5. Spearman correlation analyses of the relationships between environmental variables and the top 30 bacterial phyla (A) or classes (B). Red and blue respectively denote positive and negative correlations. ${ }^{\star} P<0.05$, ${ }^{* *} P<0.01,{ }^{* *} P<0.001$. These were done in $\mathrm{R}$ with the pheatmap package (v3.3.1, http://www.R-project.org). 


\begin{tabular}{|c|c|c|c|c|c|c|c|c|c|c|c|}
\hline $\mathbf{R}$ & Elevation & $\mathrm{pH}^{\mathrm{a}}$ & MAT & MAP & PET & $\mathrm{C} / \mathrm{N}$ & SM & SOC & TN & BGB & PSR \\
\hline Chaol index & $-0.428^{\mathrm{b} * *}$ & $0.418^{\star *}$ & $0.453^{\star *}$ & $-0.380^{\star *}$ & $0.385^{\star *}$ & -0.222 & -0.226 & -0.118 & -0.019 & $-0.270^{*}$ & -0.157 \\
\hline Shannonindex & $-0.456^{* *}$ & $0.381^{* *}$ & $0.479^{* *}$ & $-0.404^{\star *}$ & $0.437^{* *}$ & -0.179 & $-0.301^{*}$ & -0.187 & -0.077 & $-0.319^{* *}$ & -0.154 \\
\hline
\end{tabular}

Table 2. Pearson correlations (R) evaluating the association between environmental variables and bacterial diversity (Chaol richness, Shannon-Wiener diversity). ${ }^{\mathrm{a}} \mathrm{MAP}, \mathrm{MAT}, \mathrm{PET}, \mathrm{C} / \mathrm{N}, \mathrm{SM}, \mathrm{SOC}, \mathrm{TN}, \mathrm{BGB}$, and PSR respectively correspond to mean annual precipitation, mean annual temperature, evapotranspiration, carbon/ nitrogen ratio, soil moisture, total carbon, belowground biomass, and plant species richness. ${ }^{\text {b Bold values }}$ indicate significant correlations $(P<0.05)$.

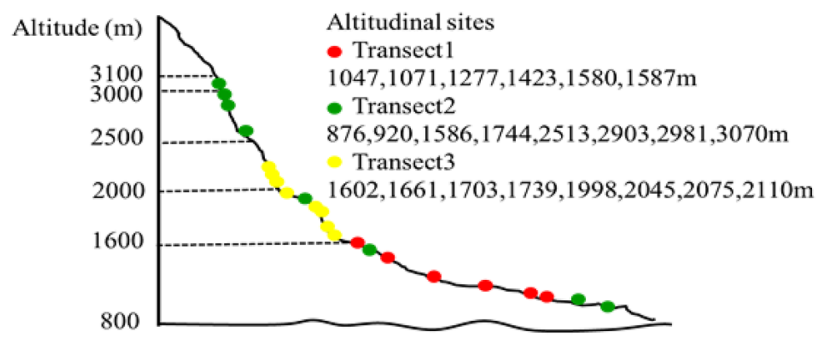

Figure 6. The locations of selected Mt. Tianshan grassland study sites along an elevation gradient. It was created using Adobe Illustrator CS6 (Adobe Software, USA).

composition and diversity of soil bacteria exhibited niche differentiation characteristics in response to difference in soil $\mathrm{pH}$, which is a primary consequence of heterogeneity in the soil environment. In conclusion, the changes in soil $\mathrm{pH}$ have a crucial role in shaping the composition and diversity of bacterial communities in the grassland ecosystem of Mt. Tianshan, China.

\section{Materials and methods}

Study region and site description. The chosen study site was situated west of Mt. Tianshan in northwest China, which is characterized by a typically arid continental temperate monsoon climate. In the present study, soil samples were collected from 22 representative sites. Each of the 22 sites was classified into three different transects (Fig. 6). There were six sites located in Transect 1 while both Transects 2 and 3 contained eight sites. Details of the sampled sites are shown in Supplementary Table 1.

Sampling design and data collection. Soil samples were collected in July 2017. Briefly, a $100 \mathrm{~m} \times 100 \mathrm{~m}$ sampling plot was established at each sampling site and the spatial geographic coordinates and elevation were recorded by using an eTrex Venture global positioning system (Garmin, USA). Three random $1 \mathrm{~m} \times 1 \mathrm{~m}$ quadrats at each site were then selected, and soil samples (depth: $0-10 \mathrm{~cm}$ ) were collected from these locations. In addition, the aboveground and belowground plant biomass from each quadrat were collected and sorted by species to compute plant species richness. After collection, samples were placed in sterile polyethylene bags, refrigerated in portable refrigeration units, and transported to the laboratory for further analysis. Soil samples were sieved through a $2.0 \mathrm{~mm}$ mesh to remove roots and other residue, and were then subdivided into two portions, one of which was maintained at $4{ }^{\circ} \mathrm{C}$ for measurements of soil properties, while the other was stored at $-80^{\circ} \mathrm{C}$ before DNA extraction.

Data regarding mean annual temperature (MAT), mean annual precipitation (MAP), and potential evapotranspiration (PET) were obtained from the WorldClim database (https://www.worldclim.org). An IQ150 pH meter (Spectrum Technologies, USA) was used to measure soil $\mathrm{pH}$ at a 1:5 soil water ratio. A gravimetric approach was used to determine soil moisture after drying for $24 \mathrm{~h}$ in a $105^{\circ} \mathrm{C}$ oven. Total nitrogen (TN) and total carbon (TC) were quantified with an Elemental Analyzer 3000 (Euro Vector, Italy). Soil inorganic carbon concentration was measured volumetrically using a Calcimeter 08.53 (Eijkelkamp, The Netherlands). The soil organic carbon levels were determined by calculating the difference between total carbon and soil inorganic carbon concentrations.

DNA extraction and 16S sequencing. A DNeasy Power Soil Kit (QIAGEN, The Netherlands) was used to extract DNA from a $1.0 \mathrm{~g}$ aliquot of each soil sample according to provided directions, after which a NanoDrop ND- 1000 instrument (Thermo Fisher Scientific, USA) and agarose gel electrophoresis, were used to evaluate DNA quantity and quality, respectively.

Research has shown that the V3-V4 region of the bacterial 16S rRNA gene provides high coverage and taxonomic accuracy with lower overestimation of alpha diversity ${ }^{25}$. Therefore, the 338F (5'-ACTCCTACGGGA GGCAGCA-3') and 806R (5'-GGACTACHVGGGTWTCTAAT-3') primers ${ }^{26}$ were used to amplify the bacterial V3-V4 region, with sample-specific 7-bp barcode sequences incorporated into the primers to facilitate multiplexed sequencing. The thermocycler settings were as follows: $98^{\circ} \mathrm{C}$ for $2 \mathrm{~min}, 25$ cycles of $98{ }^{\circ} \mathrm{C}$ for $15 \mathrm{~s}$, $55^{\circ} \mathrm{C}$ for $30 \mathrm{~s}$, and $72{ }^{\circ} \mathrm{C}$ for $30 \mathrm{~s}$, followed by $72^{\circ} \mathrm{C}$ for $5 \mathrm{~min}$. Agencourt AMPure beads (Beckman Coulter, 
USA) were used to amplify the purified PCR products, which were quantified with a PicoGreen dsDNA Assay Kit (Invitrogen, USA). Equal amounts of all amplicons were then pooled, and paired-end $2 \times 300 \mathrm{bp}$ sequencing was conducted by Shanghai Personal Biotechnology (China) with an Illumina MiSeq PE250 instrument and the MiSeq Reagent Kit v3.

Sequencing analysis. After removing the sequence adapters, the pair-end sequences were merged into full-length sequences using FLASH v1.2.3 ${ }^{27}$. Then the quantitative insights into microbial ecology QIIME pipeline (v1.8.0) was employed to process the sequencing data as previously described ${ }^{28}$. After denoising and chimera removal using DADA $2^{29}$, the remaining high-quality sequences were clustered into operational taxonomic units (OTUs) with a similarity greater than $97 \%$ using an UCLUST model ${ }^{30}$. Representative sequences for each OTU were selected by default parameters, and a BLAST search against the Greengenes database ${ }^{31}$ was used for the OTU classification of these representative sequences based upon the best match. OTU tables were generated to present OTU abundance in each sample and to detail OTU taxonomy. Those OTUs that contained fewer than $0.001 \%$ of overall sequences across all samples were discarded. To avoid bias caused by different sequencing depth, sequences were resampled to the sample sequencing depth (3167 sequences per sample) across all samples.

Bioinformatics and statistical analyses. The QIIME v1.8.0 pipelines (http://qiime.sourceforge.net) ${ }^{28}$ and R v3.3.1 packages (R Foundation for Statistical Computing; available at http://www.R-project.org) were used for sequencing data analysis. OTU-level alpha indices of alpha diversity including the Chaol index and the Shannon-Wiener index were calculated using the QIIME OTU table. Analysis of similarities (ANOSIM) was performed based on the Bray-Curtis dissimilarity by non-metric multidimensional scaling (NMDS) using the "vegan" package ${ }^{32}$. The pairwise Spearman's correlation matrix between environmental factors and bacteria was performed by the "psych" package ${ }^{33}$. Heatmap plots were generated using the "pheatmap" package (R Development Core Team, https://CRAN.R-project.org/package=pheatmap) ${ }^{34}$.

Data are given as means \pm standard error (SE) for three replicate samples. One-way analysis of variance and Duncan's multiple comparisons were used to determine the differences between treatments regarding soil physicochemical and microbial community abundance. Statistical analyses were performed using SPSS 20.0 (IBM, Corp, Armonk, NY, USA), and SigmaPlot 10.0 (Systat Software, Inc., San Jose California USA, www.systatsoft ware.com).

\section{Data availability}

All data included in this study are available upon request by contact with the corresponding author.

Received: 19 April 2021; Accepted: 13 December 2021

Published online: 07 January 2022

\section{References}

1. Nogués-Bravo, D., Araújo, M. B., Errea, M. P. \& Martinez-Rica, J. P. Exposure of global mountain systems to climate warming during the 21st Century. Glob. Environ. Change 17, 420-428 (2017).

2. Siles, J. A. \& Margesin, R. Abundance and diversity of bacterial, archaeal, and fungal communities along an altitudinal gradient in alpine forest soils: What are the driving factors?. Microb. Ecol. 72, 207-220 (2016).

3. Tang, M. et al. Elevational is the main factor controlling the soil microbial community structure in alpine tundra of the Changbai Mountain. Sci. Rep. 10, 12442 (2020).

4. Bryant, J. A. et al. Microbes on mountainsides: Contrasting elevational patterns of bacterial and plant diversity. Proc. Natl. Acad. Sci. USA 105, 11505-11511 (2008).

5. Fierer, N. et al. Microbes do not follow the elevational diversity patterns of plants and animals. Ecology 92, 797-804 (2011).

6. Nottingham, A. T. et al. Microbes follow Humboldt: Temperature drives plant and soil microbial diversity patterns from the Amazon to the Andes. Ecology 99, 2455-2466 (2018).

7. Singh, D. et al. Strong elevational trends in soil bacterial community composition on Mt. Halla, South Korea. Soil Biol. Biochem. 68, 140-149 (2014).

8. Singh, D., Takahashi, K., Kim, M., Chun, J. \& Adams, J. M. A hump-backed trend in bacterial diversity with elevation on Mount Fuji, Japan. Microb. Ecol. 63, 429-437 (2011).

9. Shen, C. et al. Soil pH drives the spatial distribution of bacterial communities along elevation on Changbai Mountain. Soil Biol. Biochem. 57, 204-211 (2013).

10. Lauber, C. L., Hamady, M., Knight, R. \& Fierer, N. Pyrosequencing-based assessment of soil pH as a predictor of soil bacterial community structure at the continental scale. Appl. Environ. Microbiol. 75, 5111-5120 (2009).

11. Nielsen, U. N., Osler, G. H., Campbell, C. D., Burslem, D. F. \& van der Wal, R. The influence of vegetation type, soil properties and precipitation on the composition of soil mite and microbial communities at the landscape scale. J. Biogeogr. 37, 1317-1328 (2010).

12. Shen, C. et al. Soil pH dominates elevational diversity pattern for bacteria in high elevation alkaline soils on the Tibetan Plateau. FEMS Microbiol. Ecol. 95, fiz003 (2019).

13. Hollister, E. B. et al. Shifts in microbial community structure along an ecological gradient of hypersaline soils and sediments. ISME J. 4, 829-838 (2010).

14. Liu, J. et al. Soil carbon content drives the biogeographical distribution of fungal communities in the black soil zone of northeast China. Soil Biol. Biochem. 83, 29-39 (2015).

15. Wang, J., Soininen, J., He, J. \& Shen, J. Phylogenetic clustering increases with elevation for microbes. Environ. Microbiol. Rep. 4, 217-226 (2012).

16. Shen, C., Ni, Y., Liang, W., Wang, J. \& Chu, H. Distinct soil bacterial communities along a small-scale elevational gradient in alpine tundra. Front. Microbiol. 6, 582 (2015).

17. Li, G. X. et al. Contrasting elevational diversity patterns for soil bacteria between two ecosystems divided by the treeline. Sci. China Life Sci. 59, 1177-1186 (2016).

18. Bahram, M., Põlme, S., Kõljalg, U., Zarre, S. \& Tedersoo, L. Regional and local patterns of ectomycorrhizal fungal diversity and community structure along an altitudinal gradient in the Hyrcanian forests of northern Iran. New Phytol. 193, 465-473 (2011). 
19. Delgado-Baquerizo, M. et al. Plant attributes explain the distribution of soil microbial communities in two contrasting regions of the globe. New Phytol. 219, 574-587 (2018).

20. Liu, S. et al. Decoupled diversity patterns in bacteria and fungi across continental forest ecosystems. Soil Biol. Biochem. 144, 107763 (2020).

21. Zeng, J. et al. Nitrogen fertilization directly affects soil bacterial diversity and indirectly affects bacterial community composition. Soil Biol. Biochem. 92, 41-49 (2016).

22. Jones, R. T. et al. A comprehensive survey of soil acidobacterial diversity using pyrosequencing and clone library analyses. ISME J. 3, 442-453 (2009).

23. Navarrete, A. A. et al. Differential response of Acidobacteria subgroups to forest-to-pasture conversion and their biogeographic patterns in the western Brazilian Amazon. Front. Microbiol. 6, 1443 (2015).

24. Shen, C. et al. Contrasting patterns and drivers of soil bacterial and fungal diversity across a mountain gradient. Environ. Microbiol. 22, 3287-3301 (2020).

25. Soriano-Lerma, R. et al. Influence of $16 \mathrm{~S}$ rRNA target region on the outcome of microbiome studies in soil and saliva samples. Sci. Rep. 10, 13637 (2020).

26. Mori, H. et al. Design and experimental application of a novel non-degenerate universal primer set that amplifies prokaryotic 16s rRNA genes with a low possibility to amplify eukaryotic rRNA genes. DNA Res. 21, 217-227 (2013).

27. Magoč, T. et al. FLASH: Fast length adjustment of short reads to improve genome assemblies. Bioinformatics 27, 2957-2963 (2011).

28. Caporaso, J. G. et al. QIIME allows analysis of high-throughput community sequencing data. Nat. Methods 7, 335-336 (2010).

29. Callahan, B. J. et al. DADA2: High-resolution sample inference from Illumina amplicon data. Nat. Methods 13, 581-583 (2016).

30. Edgar, R. C. Search and clustering orders of magnitude faster than BLAST. Bioinformatics 26, 2460-2461 (2010).

31. DeSantis, T. Z. et al. Greengenes, a chimera-checked $16 \mathrm{~S}$ rRNA gene database and workbench compatible with ARB. Appl. Environ. Microbiol. 72, 5069-5072 (2006).

32. Oksanen, J. et al. Vegan: Community Ecology Package (R package version 2.2-0) (2014).

33. Revelle, W. Psych: Procedures for Personality and Psychological Research, Northwestern University, Evanston, Illinois, USA, https://CRAN.R-project.org/package=psych Version $=1.8 .10(2018)$.

34. Kolde, R. et al. Package 'pheatmap'. R package 1, 790 (2015).

\section{Acknowledgements}

We thank students from Xinjiang Agriculture University for their field assistance in surveying community composition.

\section{Author contributions}

R.L. wrote the first draft. R.L. and P.Z. provided experimental determination. Y.H.L. provided on the analysis approach. J.H.C. guidance on statistical analysis. N.N.X. wrote the response letter. Z.J.S., N.L., X.S.D., W.H.F., J.D., Y.C.M. and M.F.L. participate in field sampling and testing. J.D.S. conceived the experiment designed. All authors contributed to improve the final manuscript.

\section{Funding}

This work was supported jointly by the National Natural Science Foundation of China (U160320014), Grassland Peak Discipline Foundation of Xinjiang Uygur Autonomous Region, China (CXGFXK-2018-06) and Graduate innovation projects of Xinjiang Uygur Autonomous Region, China (XJ2019G140).

\section{Competing interests}

The authors declare no competing interests.

\section{Additional information}

Supplementary Information The online version contains supplementary material available at https://doi.org/ 10.1038/s41598-021-03937-x.

Correspondence and requests for materials should be addressed to J.S.

Reprints and permissions information is available at www.nature.com/reprints.

Publisher's note Springer Nature remains neutral with regard to jurisdictional claims in published maps and institutional affiliations.

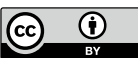

Open Access This article is licensed under a Creative Commons Attribution 4.0 International License, which permits use, sharing, adaptation, distribution and reproduction in any medium or format, as long as you give appropriate credit to the original author(s) and the source, provide a link to the Creative Commons licence, and indicate if changes were made. The images or other third party material in this article are included in the article's Creative Commons licence, unless indicated otherwise in a credit line to the material. If material is not included in the article's Creative Commons licence and your intended use is not permitted by statutory regulation or exceeds the permitted use, you will need to obtain permission directly from the copyright holder. To view a copy of this licence, visit http://creativecommons.org/licenses/by/4.0/.

(C) The Author(s) 2022 